\title{
VERIFICATION OF CONJUGATE HEAT TRANSFER MODELS IN A CLOSED VOLUME WITH RADIATIVE HEAT SOURCE
}

\author{
Vyacheslav I. Maksimov ${ }^{1,}{ }^{*}$, Tatiana A. Nagornova ${ }^{1}$, and Nikolai I. Kurilenko ${ }^{2}$ \\ ${ }^{1}$ National research Tomsk Polytechnic University, 634050 Tomsk, Russia \\ ${ }^{2}$ Tyumen State Architecture - and Construction University, 625001 Tyumen, Russia
}

\begin{abstract}
The results of verification of mathematical model of convective-conductive heat transfer in a closed volume with a thermally conductive enclosing structures are presented. Experiments were carried out to determine the temperature of floor premises in the working conditions of radiant heating systems. Comparison of mathematical modelling of temperature fields and experiments showed their good agreement. It is concluded that the mathematical model of conjugate heat transfers in the air cavity with a heat-conducting and heat-retaining walls correspond to the real process of formation of temperature fields in premises with gas infrared heaters system.
\end{abstract}

\section{Introduction}

The tasks of free convection in the closed volumes attract attention of many researchers in the recent decades. A large part of research is devoted to the processes of heat transfer without regard to the heat sink in the building envelope (eg [1-3]). To the tasks of Conjugate heat transfer is given much less attention (for example 4, 5) due to the problems of energy equations solution in the area with discontinuities transfer coefficient. The tasks of free convection in the regions with the heat-conducting walls and the heat-storing enclosing constructions with local radiant heating sources are even more complex [6-8]. The solutions [6-8] are obtained for the conditions of complex heat exchange - due to emitter energy, located in the upper part of solution region, when heated a thin near-surface layer of the lower part of the enclosing rectangular constructions with the subsequent warming up of air as a result of free convection under the conditions of heat sink in the vertical walls. But results [6-8] did not pass the verification stage according to the experimental data due to problems of the organization of experimental studies at the necessary for the comparison level of the metrological guarantee of temperature measurements.

The purpose of this research - the experimental and theoretical study of the processes conjugate heat transfer in a closed rectangular premises with a local radiant heat source and heat-conducting walls under identical conditions. The main objective is to verify the

\footnotetext{
* Corresponding author: elf@tpu.ru
} 
mathematical formulation [6-8] on the results of experiments on large-scale models of typical objects with radiant heating systems.

\section{The methodology of the research and experimental results}

Research on measuring temperatures in typical sections held indoors (fig. 1) with a gas infrared emitter (Russian manufacture "Termoshvak") located in the upper part of the analysis region. Power of emitter $-30 \mathrm{~kW}$. On the concrete base, starting from the axis of symmetry of the emitter in the directions $X$ and $Y$ (fig. 1) were established thermocouples at a distance $300 \mathrm{~mm}$ from each other.

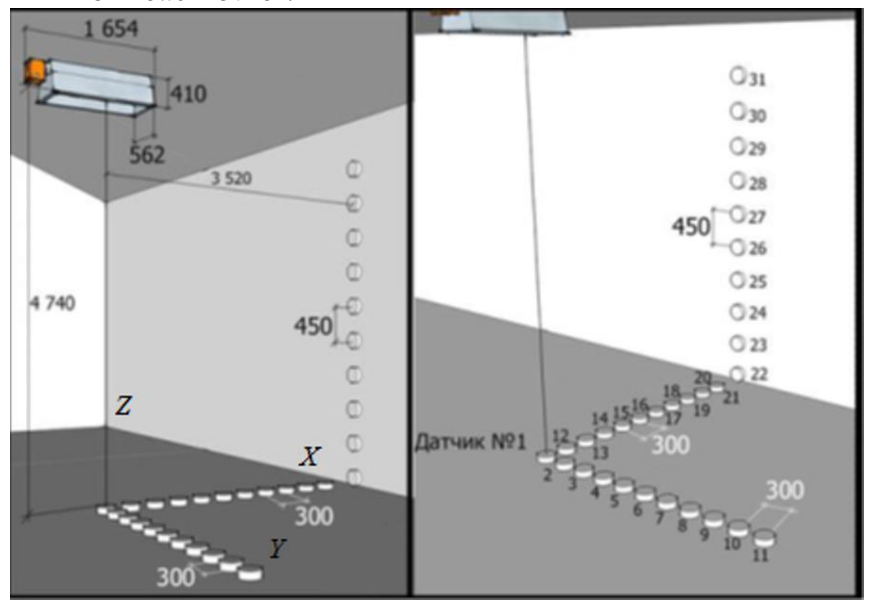

Fig. 1. The area of experimental studies and sensor layout: dimensions in $\mathrm{mm}$.

The studies were conducted in winter period when the outside air temperature minus $20{ }^{\circ} \mathrm{C}$. At the initial time the indoor temperature $18{ }^{\circ} \mathrm{C}$. Dimensions of emitter $1654 \times 410$ $\mathrm{mm}$. The vertical enclosing constructions are made from sandwich- panels with the known thermophysical characteristics. Temperature measurements were made in the floor surface during a time interval corresponding to the output of the thermal regime close to a stationary ( 1 hour) using of thermocouple and optical pyrometer with systematic errors $\pm 4 \%$. As a results of experiment were obtained values of heat flux to the surface of concrete base (table).

Table. Heat flux distribution along the coordinate $\mathrm{x}$ of the section passing through the axis of GIE symmetry to the right side enclosing structure (fig. 1).

\begin{tabular}{|l|c|c|c|c|c|c|c|c|c|}
\hline $\begin{array}{l}\text { coordinate, } \mathrm{m} \\
\text { (fig. 1) }\end{array}$ & 0 & 0.5 & 1 & 1.5 & 2 & 2.5 & 3 & 3.5 & 4 \\
\hline$q, \mathrm{~W} / \mathrm{m}^{2}$ & 190.5 & 180.2 & 182.1 & 172.2 & 134.2 & 122.2 & 102.1 & 106 & 80.2 \\
\hline
\end{tabular}

It was found that the proportion of thermal energy from the emitter to the floor surface, close to $57 \%$ of the total allocated heat of GIE. It should be noted that the temperature of the concrete floor surface is selected as the object of measurements due to high heatretaining capacity of concrete. It is substance with the thermophysical characteristics sufficiently uniform in the volume. Therefore the process of heating concrete floor is sufficiently inertia and improbable the random deviations of temperatures in individual sections of surface. 
Fig. 2 shows the results of experiments in the form of a floor surface temperature distributions $\left(T_{f}\right)$ in the direction $X$.

Distribution of $T_{f}$ on $X$ shows its nonmonotonic variation with distance from the point corresponding to the symmetry axis of the GIE.

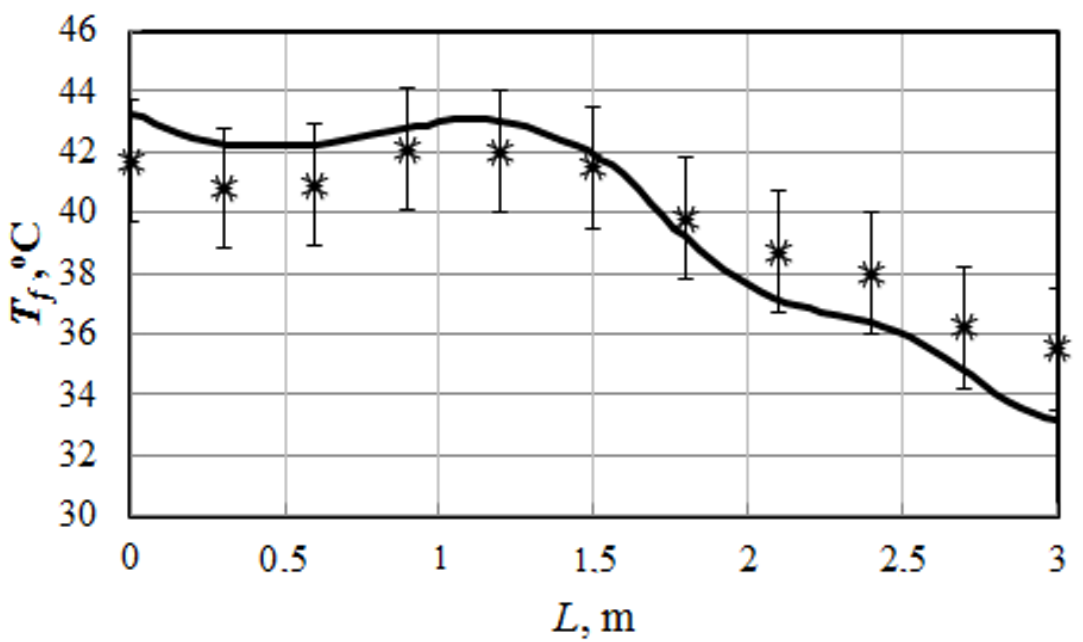

Fig. 2. The distribution of the floor surface temperature of the transmitter center to the side walling: * - experimental results with a confidence interval; solid line - the results of mathematical modeling.

It is clearly seen that at a distance of $3 \mathrm{~m}$ from the axis of symmetry the maximum difference $T_{f}$ is 10 degrees. The character of changes $T_{f}$ with increasing $X$ complies with the conditions of the inhomogeneous heat transfer at the "concrete - air" section. Such distribution $T_{f}(X)$ can only be the result of natural convection processes, due to the heating of the concrete to temperatures more $33^{\circ} \mathrm{C}$. One can reasonably conclude about shaping of vortex cells in influence zone of infrared radiation on the surface concrete floor.

\section{Numerical investigations}

Mathematical modelling of heat transfer processes carried out for the area shown in fig. 3 . The dimensions of the solutions field $8 \times 6 \mathrm{~m}$.

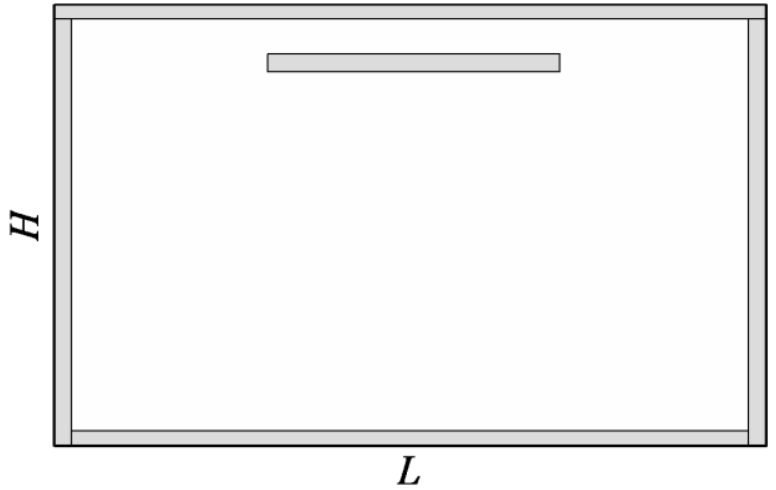

Fig. 3. Area of solving the problem: $L, H$ - the height and length of solutions area respectively.

Statement of the Problem [6] accounted for convective heat transfer in the air and conductive in enclosing structures. For all the external borders of the area to account the 
convective and radiative heat exchange with the environment. On the left and right boundaries (fig. 3) is outside air with temperature minus $20^{\circ} \mathrm{C}$.

On the surface of the GII was set established in the experiment the surface temperature of the radiator "Tehnoshvak - 20". On three internal interfaces of media the boundary conditions of the fourth kind are assigned (equality of heat fluxes and temperatures). On the lower concrete base was assigned the value of heat flux, which varied depending on coordinate $q(X)$, on the basis obtained experimental data (table). Initial conditions started as the analogous to initial data of experimental studies.

For describing the process of the combined heat transfer in the solution region (fig. 3) as the base was used mathematical model [6-8], developed for calculating the heat transfer in the locked air region with the heat-conducting enclosing walls and the local energy source.

System of equations, which describe the process of heat transfer in this conditions, includes the Navier-Stokes equations, recorded in the transformed variables (function of current - vorticity) [4-8], and the equations of energy for each of the region elements (fig. 3 ). For the variables the function of current and vorticity on the interfaces of media were written analogous [4-8] boundary conditions.

The system of partial differential equations is solved by the finite-difference method $[4,5]$ with the use of an algorithm [4, 5], that developed for the numerical solution of free convection in the locked rectangular regions with the local energy sources.

\section{Results and discussion}

As a result the solutions of the problem of convective- conductive heat transfer are obtained temperature fields and the contour lines of the current function, which describe the processes being investigated. In fig. 4 are represented the results of the numerical simulation, which illustrate basic laws governing the heat transfer in the considered region with the heating system on the base GIE for moment of time, which corresponds to experiment (1 hour).

It is established that near GIE are reached sufficiently high temperatures of air (to 200 $\left.{ }^{\circ} \mathrm{C}\right)$. But at a distance the half meter from it of temperature considerably they are lowered. In the section $Z=4.5 \mathrm{~m} T$ it changes insignificantly: in the range from 32 to $35^{\circ} \mathrm{C}$. And only on the concrete base $T=40$ (fig. 2 and $4 a$ ). Above GIE also are formed the zones of high temperature (to $110^{\circ} \mathrm{C}$ ). With the minimally permissible distance of emitter from the surface of upper floors are formed the flow of heated air of the type of Benard cells (fig. 4 $b$ ).

In fig. 2 are represented the results of experimental and numerical studies in the form of the temperature distributions on the surface of concrete floor in the direction $\mathrm{X}$ from the center section of the region to right side vertical wall. In the figure the confidence interval of the measurements of temperatures, which composed $\pm 2{ }^{\circ} \mathrm{C}$, is also noted. The results of mathematical simulation fall into this interval.

It is possible to make a conclusion about the good agreement of the obtained with the use of a mathematical model 6-8 of theoretical consequences and experimental values of the fundamental characteristic of the process -the temperature of floor surface. With the analysis of laws governing heating air under the conditions in question it is necessary to have in the form that the energy, which comes from emitter, is not absorbed by air, and accumulates in the thin near-surface layer of concrete floor.

Subsequently the concrete heats the air (arriving flying to the surface of floor), which rises upward, freeing the zone of intensive heating for cold air, which is descended. Thus, is formed the circulation flow, whose typical example is given in fig. 4 . 


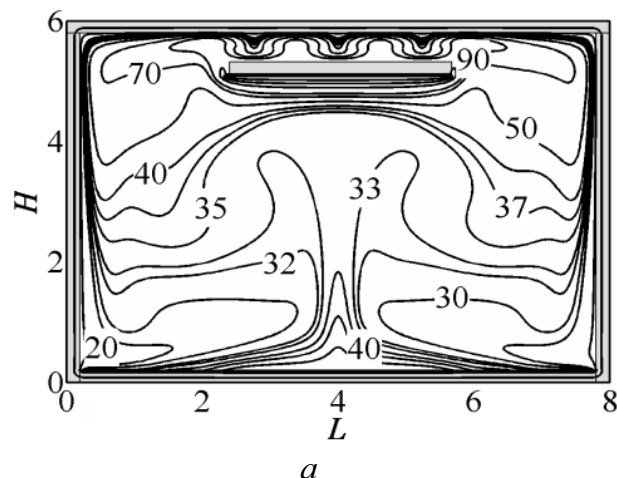

Fig. 4. The temperature distribution $(a)$ and current functions $(b)$ at time 1 hour. Values the temperature is listed in ${ }^{\circ} \mathrm{C}$.

In conclusion, it should be noted that the model [6-8], as demonstrated by its verification by comparison with the results of the temperature measurements in an environment adequate accepted in mathematical modeling, has a good potential for development in terms of a more detailed description of the temperature field of emitter. This level of detail can create the preconditions for a more accurate calculation of air temperature and the corresponding specification of the parameters of the microclimate of industrial premises with heating systems based on gas infrared emitters.

\section{Conclusion}

Verification of the mathematical model of heat transfer process of closed volume with thermally conductive in enclosing structures showed good agreement between the experimental results and the previously developed [6-8] mathematical model of convectiveconductive heat transfer in a closed system under consideration with radiant heating.

\section{Acknowledgments}

The work was supported by the Russian President's grant (Scientific School project 7538.2016.8).

\section{References}

1. P. Kumar, V. Eswaran, Int. J. Therm. Sc. 67 (2013)

2. K. Lari , M. Baneshi , S.A. Gandjalikhan Nassab, A.S. Komiya, Int. J. Heat Mass Trasf. 54 (2011)

3. R. Roslan, H. Saleh, I. Hashim, A.S. Bataineh, Natural convection in an enclosure containing a sinusoidally heated cylindrical source, Int. J. Heat Mass Trasf. 70 (2014)

4. G.V. Kuznetsov, M.A. Sheremet, Fl. Dyn. 41 (2006)

5. G.V. Kuznetsov, M.A. Sheremet, Int. J. Heat Mass Transf. 54 (2011)

6. G.V. Kuznetsov, N.I. Kurilenko, V.I. Maksimov, G.Ya. Mamontov, T.A. Nagornova, JEPT 86 (2013)

7. T.A. Nagornova, Chem. Petr. Eng. 51 (July 2015)

8. V.I. Maksimov, T.A. Nagornova, EPJ Web Conf. 76 (2014) 